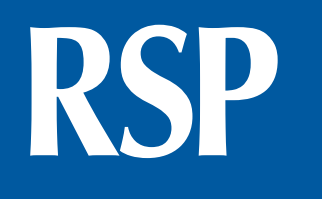

http://www.rsp.fsp.usp.br/
Revista de Saúde Pública

\title{
Efetividade de uma intervenção digital na diminuição da prática de binge drinking entre frequentadores de baladas
}

\author{
Yago C Baldin', Adriana Sanudo', Zila M Sanchez' \\ ' Universidade Federal de São Paulo. Escola Paulista de Medicina. Departamento de Medicina Preventiva. \\ São Paulo, SP, Brasil
}

\section{RESUMO}

OBJETIVO: Avaliar, após seis meses, a efetividade de uma intervenção digital na diminuição da prática de binge drinking entre frequentadores de baladas.

MÉTODOS: Foi realizado um inquérito de portal através de uma amostra probabilística em 31 baladas da cidade de São Paulo, a qual originou um ensaio controlado randomizado com 1.057 participantes. Aqueles classificados como uso problemático do álcool $(\mathrm{n}=465)$ através do Alcohol Use Disorders Identification Test foram randomizados em dois grupos de estudo - intervenção e controle. A intervenção digital consistiu em expor os participantes a uma tela de feedback normativo sobre seu consumo de álcool, caracterizando os riscos associados à quantidade consumida, valores gastos com bebidas, beber e dirigir, classificação do risco do Alcohol Use Disorders Identification Test e dicas de redução de danos.

RESULTADOS: Houve uma redução significativa da prática de binge drinking na semana estimada em $38 \%$ entre os participantes do grupo intervenção após seis meses $(\mathrm{p}<0,05)$. Porém, não houve redução significativa nos desfechos quando se analisam os grupos intervenção e controle e os tempos início e seis meses após, simultaneamente.

CONCLUSÕES: Não se pode concluir que as ferramentas digitais reduzem o padrão de binge drinking nos frequentadores de baladas paulistanas. São necessários mais estudos com esta metodologia devido ao caráter atrativo para esse grupo, tendo em vista a privacidade e a rapidez que as informações personalizadas são transmitidas.

DESCRITORES: Bebedeira, prevenção \& controle. Internet. Avaliação de Eficácia-Efetividade de Intervenções. Ensaio Controlado Aleatório.

Recebido: 20 set 2016

Aprovado: 20 dez 2016

Como citar: Baldin YC, Sanudo A, Sanchez ZM. Efetividade de uma intervenção digital na diminuição da prática de binge drinking entre frequentadores de baladas. Rev Saude Publica. 2018;52:2.

Copyright: Este é um artigo de acesso aberto distribuído sob os termos da Licença de Atribuição Creative Commons, que permite uso irrestrito, distribuição e reprodução em qualquer meio, desde que o autor e a fonte originais sejam creditados. 


\section{INTRODUÇÃO}

As casas noturnas são um importante local de lazer e de entretenimento para os jovens ${ }^{1}$. O consumo abusivo de álcool e o ambiente onde as quebras de padrões sociais são toleradas e o prazer é estimulado ${ }^{2}$ contribuem para a maior exposição a riscos dos frequentadores de baladas, como violência física ou sexual ${ }^{3}$, agressões e violações de conduta ${ }^{4}$. Na balada, é comum a realização do binge drinking (BD) $)^{5}$, ou seja, o consumo de no mínimo quatro doses de álcool em uma única ocasião para mulheres e cinco doses para homens ${ }^{6}$. Essa prática pode aumentar a chance de consequências danosas do abuso do álcool ${ }^{7}$, pois está associada a maiores chances de abuso sexual, tentativas de suicídio, sexo desprotegido, gravidez indesejada, embriaguez, quedas, acidentes e doenças inflamatórias ${ }^{8}$.

Para diminuição do consumo de álcool e outras drogas, recentemente intervenções digitais (i.e., ofertadas através da internet) vêm sendo testadas em países desenvolvidos, especialmente entre jovens ou estudantes ${ }^{9,10}$, por meio do uso de mensagens de feedback normativo personalizado ${ }^{11,12}$. As mensagens personalizadas abordam informações didáticas sobre uso do álcool, mensagens pessoais sobre o perfil de beber do participante, seus fatores de risco e consequências danosas, custos associados ao consumo, comparações com outros perfis e dicas de redução de danos ${ }^{13,14}$ confrontando o consumo individual com o da população ou fornecendo formas sobre como mudar seu comportamento ${ }^{15}$. Esse tipo de ferramenta tem a vantagem de poder ser utilizada em grande escala por ser de fácil acesso e baixo custo ${ }^{16}$, além de respeitar a privacidade dos participantes ${ }^{17}$.

Estudos internacionais mostraram redução significativa da prática do BD em estudantes universitários após seis meses da utilização do feedback normativo personalizado via web ${ }^{18,19}$. Kypri et al. observaram, após seis meses da intervenção, uma redução significativa de $11 \%$ no volume total consumido de álcool, diminuindo problemas pessoais, sexuais e legais relacionados ao álcool, e uma redução significativa de 19\% em relação a problemas acadêmicos, como deixar de cumprir tarefas por conta do consumo de álcool ${ }^{20,21}$.

No Brasil, apesar de haver amplo acesso à internet ${ }^{22}$, as intervenções digitais com a finalidade de reduzir o abuso de álcool em nossa população vêm sendo utilizadas apenas recentemente ${ }^{23}$, mesmo sendo clara a necessidade de se implantar esse tipo de ferramenta no país, após adaptações que levem em conta diferenças culturais ${ }^{24}$. Por esse motivo, introduzir intervenções entre frequentadores de balada para reduzir problemas relacionados ao BD é um campo de atuação necessário e promissor ${ }^{25}$, tornando o presente estudo inovador ao testar essas intervenções entre frequentadores de baladas. Assim, o objetivo deste estudo foi o de avaliar, após seis meses, a efetividade de uma intervenção digital, via internet, na diminuição da prática de BD e falta de controle sobre a quantidade de álcool, entre frequentadores de balada.

\section{MÉTODOS}

\section{Amostra do Inquérito de Portal}

Os dados utilizados neste trabalho tiveram origem em um inquérito de portal ${ }^{26}$ realizado com a finalidade de diagnosticar o consumo de drogas e outros comportamentos de risco entre frequentadores de baladas da cidade de São Paulo em $2013^{27-29}$. Neste estudo, baladas foram definidas como um estabelecimento que apresentava controle de entrada e saída, venda de bebidas alcóolicas e pista de dança. Detalhes da amostragem do estudo transversal são encontrados em Sanchez et al..$^{28}$

\section{Coleta de Dados, Instrumentos e Variáveis}

A Figura 1 apresenta o fluxograma para recrutamento de frequentadores de baladas, coleta de dados e participação na intervenção, desde o início do inquérito de portal até os seis meses de acompanhamento. Os indivíduos selecionados nas filas das baladas participaram do estudo em três etapas ${ }^{26}: 1$ ) em entrevista presencial na entrada e saída da casa noturna, 2) respondendo a um questionário via internet, no dia seguinte,3) participação no ensaio controlado randomizado(ECR). 
Na fila de entrada da balada, foram abordados sistematicamente 3.063 sujeitos em 31 baladas, de maneira que o último de cada três indivíduos era convidado a participar do projeto. Os critérios de inclusão utilizados foram: pretender entrar na balada e ter pelo menos 18 anos de idade. No caso de recusa, dados sobre idade e gênero eram registrados e a próxima pessoa era abordada. Concordaram em participar da pesquisa 2.422 sujeitos (aceite de 79\%), que responderam a uma entrevista sobre variáveis sociodemográficas, padrões de consumo de álcool e comportamentos de risco em baladas nos 12 meses anteriores à entrevista $\mathrm{e}$ tiveram sua concentração de álcool no ar exalado mensurada no momento da entrevista por meio de um etilômetro.

Na saída da balada, aqueles que haviam sido entrevistados na entrada (identificados via pulseira com código numérico único) foram solicitados a responder a uma nova entrevista. Depois, era entregue um folder com informações sobre a participação em uma pesquisa on-line.

No dia seguinte ao recrutamento, o link de um questionário pós-balada, subdividido em dois módulos, foi enviado, pelo correio eletrônico, aos 1.833 participantes da entrevista na saída das baladas. Desses, 1.222 acessaram o questionário (aceite de 67\%). O questionário continha questões referentes aos comportamentos de risco dos participantes realizados após saída da balada, gastos com álcool, maior quantidade de doses consumida, tempo de consumo, tipo de bebida e a avaliação do Alcohol Use Disorders Identification Test (AUDIT), com 10 questões que produzem um escore variando de zero a 40 e que os classifica em quatro níveis de risco o padrão de consumo de álcool (uso de baixo risco; uso de risco; uso nocivo; dependência).

O AUDIT foi desenvolvido pela Organização Mundial da Saúde (OMS) para identificar frequência, quantidade e consequências do abuso de álcool ${ }^{30}$. Como instrumento de avaliação, o AUDIT tem mostrado um número crescente de evidências como uma metodologia rápida e de sucesso na identificação desses padrões ${ }^{31}$. No presente estudo, o AUDIT foi utilizado para identificar os padrões de consumo de álcool entre frequentadores de baladas e selecioná-los para um ECR de uma intervenção digital com feedback normativo personalizado.

Neste estudo, planejou-se análise das seguintes variáveis de desfecho: binge drinking no mês $\left(\mathrm{BD}_{\text {mês }}\right)$, binge drinking na semana $\left(\mathrm{BD}_{\text {semana }}\right)$ e falta de controle sobre o comportamento de beber (falta de controle), sendo as duas últimas advindas do AUDIT e a primeira, uma questão extra.

$\mathrm{O} \mathrm{BD}_{\text {mês }}$ refere-se à seguinte pergunta (extra-AUDIT): "Nas últimas quatro semanas, qual foi a maior quantidade de doses padrão de álcool que você consumiu em uma única ocasião?". Essa era uma questão aberta e, para fins de análise, foi categorizada em "sim" quando a resposta foi cinco ou mais doses e "não" para aquelas que foram menores do que cinco.

$\mathrm{O} \mathrm{BD}_{\text {semana }}$ refere-se à pergunta três do AUDIT: "Com que frequência você consome cinco ou mais doses de bebidas em uma única ocasião?”. Para fins de análise, categorizou-se como "sim" as respostas "Pelo menos uma vez por semana (semanalmente)" e "Diariamente ou quase diariamente" e como "não", "Nunca", "Menos de uma vez por mês" e "Pelo menos uma vez por mês (mensalmente)". Destaca-se que, para essa pergunta, optou-se por utilizar-se a opção de cinco doses com base em uma concentração média de $12 \mathrm{~g}$ de etanol por dose, seguindo a orientação original do AUDIT que pressupõe, para essa questão, $60 \mathrm{~g}$ de etanol ou mais. Assim, as opções de dose apresentadas ao participante foram de $330 \mathrm{ml}$ de cerveja (1 lata; 4,7\% etanol / $130 \mathrm{ml}$ de vinho; $12 \%$ de etanol / $40 \mathrm{ml}$ de destilado; 39\% etanol).

Falta de controle sobre o comportamento de beber refere-se à pergunta quatro do AUDIT "Nos últimos 12 meses, com que frequência você percebeu que não conseguia parar de beber depois de ter começado?". Para fins de análise, categorizou-se como "sim" as respostas "Menos de uma vez por mês", "Pelo menos uma vez por mês (mensalmente)", "Pelo menos uma vez por semana (semanalmente)" e "Diariamente ou quase diariamente" e como "não", as respostas "Nunca". 
Os dados sociodemográficos foram retirados do banco de dados inicial, ou seja, aquele referente às entrevistas cara a cara feitas na entrada das baladas, como descrito em Santos et al. ${ }^{32}$ As variáveis sociodemográficas de ajuste utilizadas foram: sexo (masculino; feminino), idade e status socioeconômico, obtido da classificação da Associação Brasileira de Estudos Populacionais (ABEP, 2012) ${ }^{33}$ de nível socioeconômico (A = alta; $\mathrm{B}$ = média-alta; $\mathrm{C}$ = média; $\mathrm{D}$ = média-baixa; $\mathrm{E}$ = baixa; sendo $\mathrm{C}, \mathrm{D}$ e $\mathrm{E}$ agrupadas pela pequena quantidade de amostra).

\section{Procedimentos do Ensaio Controlado Randomizado}

Dos 1.222 frequentadores de baladas que acessaram o questionário on-line, um total de 1.057 havia consumido álcool no último ano e concordou em participar (aceite de 86,5\%) do ECR. A alocação nos grupos foi aleatória e estratificada, realizada no próprio site, por meio de algoritmo para randomização permutada em blocos estratificados (stratified permuted block randomization), considerando as seguintes categorias para cada um dos três estratos de randomização: 1) sexo (feminino, masculino); 2) faixa etária (18-24; 25-34; 35+) e 3) padrão de consumo de álcool (classificação do AUDIT em quatro categorias, ou seja, 0-7; 8-14; 15-19; 20-40 pontos). Ao final da randomização, obteve-se o grupo intervenção com $515(48,7 \%)$ participantes e o controle com 542 (51,3\%).

A intervenção digital de feedback normativo personalizado foi aplicada em um único momento, após a avaliação inicial, e seguiu o modelo adotado por Kypri et al..$^{19}$ Os sujeitos do grupo intervenção foram expostos a uma tela de feedback normativo personalizado sobre os riscos associados ao consumo de álcool, composto de quatro partes: 1) feedback sobre o nível de consumo de álcool na ocasião investigada com informações padronizadas para cada nível de risco (saúde orgânica e mental, além de complicações sociais); 2) informações sobre as normas sociais com apresentação de gráficos de barras do percentual de brasileiros, na mesma faixa etária e sexo, que relataram beber menos álcool, enfatizando o quanto aquele comportamento é atípico para a população geral (dados da população geral do levantamento domiciliar sobre álcool de $2006^{34}$ ); 3) estimativa personalizada dos gastos financeiros por mês e ano com o álcool; e 4) informações gerais com dados para minimizar as consequências adversas do consumo de álcool. Participantes do grupo controle não receberam nenhuma intervenção e apenas responderam ao questionário.

Neste estudo, foram incluídos participantes com pontuação maior ou igual a oito no AUDIT, ou seja, aqueles classificados pelo AUDIT como tendo uso problemático do álcool (escore AUDIT $\geq 8$ ). Dos 1.057 participantes randomizados nos dois grupos, 465 (44,0\%) preencheram o critério de inclusão.

Seis meses após a resposta inicial, todos os 465 participantes foram contatados por correio eletrônico contendo o link de um novo questionário para ser preenchido para uma nova avaliação. No caso de não responderem ao questionário no período de três dias, novo link era enviado, além de SMS, avisando sobre o correio eletrônico. Após três tentativas de envio do correio eletrônico, sem resposta, o participante foi contatado por telefone e avisado sobre o link enviado por correio eletrônico. A logística de envio de correios eletrônicos, SMS e posterior ligação telefônica foi a mesma utilizada nos diferentes tempos do estudo.

\section{Análise Estatística}

Variáveis qualitativas foram descritas como número (n) e porcentagem (\%), ao passo que as quantitativas como média e desvio padrão. Associação entre variáveis qualitativas foi avaliada pelo teste qui-quadrado de Pearson ou teste Exato de Fisher quando um ou mais valores esperados eram menores do que cinco. Já a comparação de variáveis quantitativas de acordo com uma variável qualitativa dicotômica, foi realizada pelo teste t de Student.

As variáveis de desfecho foram analisadas por modelos lineares generalizados (Generalized Linear Models) com dados de painel por meio do procedimento "xtlogit" (Stata/SE 13.1 for Windows - StataCorp). Todos os modelos incluíram efeito de grupo (intervenção ou controle), tempo (momento inicial ou seis meses de acompanhamento) e interação entre grupo e 
tempo. O termo de interação permitiu avaliar o efeito da intervenção entre as duas avaliações. Os resultados foram apresentados como odds ratio bruto $\left(\mathrm{OR}_{\mathrm{b}}\right)$ e respectivo intervalo de confiança de 95\% (IC95\%). Além desse modelo, foi ajustado um novo modelo para cada uma das variáveis de desfecho, levando em consideração, além dos parâmetros já descritos, o sexo, a idade e a classe socioeconômica. Esses resultados foram expressos como odds ratio ajustado $\left(\mathrm{OR}_{\mathrm{aj}}\right)$ e respectivos IC95\%. Os preditores foram considerados fatores fixos nos modelos.

Foi realizada a comparação dos dados referentes ao sexo, idade, classe socioeconômica, escore AUDIT no tempo inicial e grupo de alocação entre os participantes que responderam o acompanhamento de seis meses, versus aqueles que não responderam, para verificar se os dados dos respondentes permaneciam homogêneos com relação à amostra inicial e também para mostrar se a não resposta ao acompanhamento foi diferente de acordo com o grupo de randomização.

Os participantes foram analisados no grupo para os quais eles foram randomizados ao início, na chamada análise por intenção de tratar (ITT - intention to treat analysis). Também foi realizada uma reanálise dos dados por meio de uma imputação utilizando o método LOCF (Last Observed Carried Forward), ou seja, para os participantes que não responderam ao acompanhamento de seis meses, considerou-se a sua resposta no momento inicial como sendo também a resposta no acompanhamento de seis meses.

Em toda a análise estatística, foi adotado um nível de significância de 5\%.

\section{Ética}

O estudo foi aprovado pelo Comitê de Ética de Pesquisa da Universidade Federal de São Paulo (Processo 21477), conduzido nos anos de 2013 e 2014 e registrado no REBEC (Registros Brasileiros de Ensaios Clínicos, do Ministério da Saúde), sob o número RBR-35bkzc.

\section{RESULTADOS}

Dos 1.832 frequentadores de baladas convidados a responder a um questionário on-line enviado por correio eletrônico, 610 (33,3\%) não o responderam (Figura 1). Desses, 1.057 $(86,5 \%)$ aceitaram participar do ECR, dos quais 465 (44,0\%) apresentaram escore AUDIT maior ou igual oito - "uso problemático de álcool" -, sendo então randomizados em dois grupos: intervenção $(n=224)$ e controle $(n=241)$.

Assim, 465 frequentadores de baladas (44,0\%; IC95\% 41,0-47,0) foram classificados como "uso problemático de álcool”. Desses, 344 (74,0\%; IC95\% 69,7-77,9) e 256 (55,2\%; IC95\% 50,5-59,7) apresentaram resposta "sim" para $\mathrm{BD}_{\text {mês }}$ e $\mathrm{BD}_{\text {semana }}$, respectivamente. Aproximadamente $50 \%$ desses frequentadores com uso problemático de álcool relataram falta de controle sobre o comportamento de beber ( $\mathrm{n}=231$; IC95\% 45,1-54,4).

De acordo com a Tabela 1, os grupos intervenção e controle mostraram-se homogêneos quanto às variáveis idade $(\mathrm{p}=0,237)$, sexo $(\mathrm{p}=0,099)$, status socioeconômico $\mathrm{ABEP}(\mathrm{p}=0,852)$ e escore AUDIT ( $p=0,332)$.

No acompanhamento de seis meses, obteve-se resposta de 79 (50,6\%) participantes do grupo intervenção e 77 (49,4\%) participantes do grupo controle. A Tabela 2 apresenta os resultados de atrito (attrition) dos dados sociodemográficas, escore AUDIT do momento inicial e grupo de alocação entre esses 156 participantes que responderam versus aqueles sem resposta. Não houve diferença estatisticamente significante entre os participantes que responderam ao questionário em seis meses versus aqueles que não responderam.

A Tabela 3 apresenta as medidas-resumo para os desfechos avaliados no tempo inicial e em seis meses de acompanhamento, de acordo com o grupo. Pode-se observar que não houve diferença significativa entre os dois grupos em nenhum dos dois momentos de avaliação. 


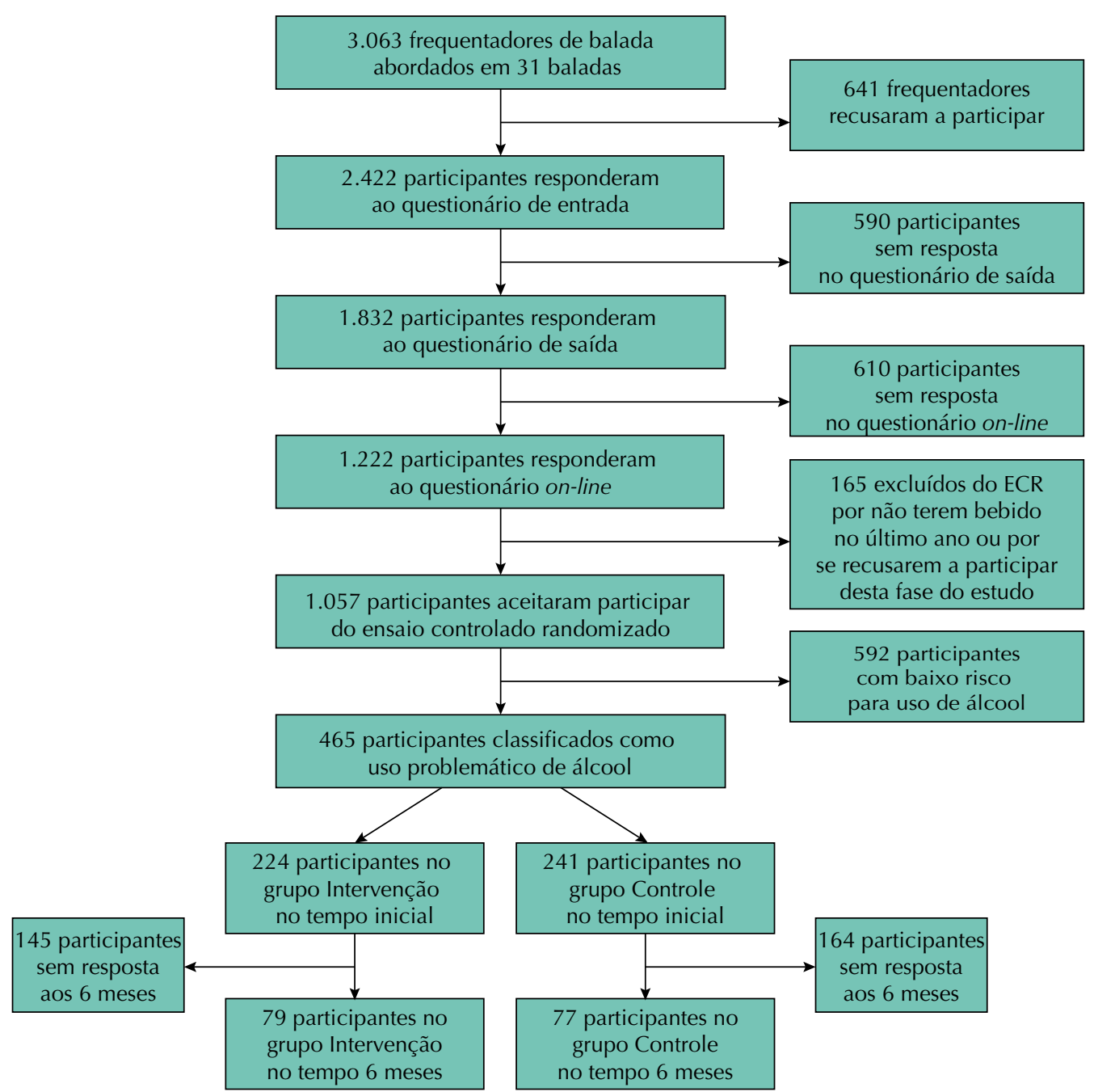

ECR: ensaio controlado randomizado

Figura 1. Fluxograma para recrutamento de frequentadores de baladas e participação no ensaio controlado randomizado para avaliação de intervenção digital com feedback normativo para redução de abuso de álcool. São Paulo, Brasil, 2013.

Tabela 1. Distribuição dos 465 participantes de acordo com grupo, dados sociodemográficos e escore AUDIT no tempo inicial.

\begin{tabular}{|c|c|c|c|c|c|c|c|c|}
\hline \multirow{3}{*}{ Variável } & \multirow{2}{*}{\multicolumn{2}{|c|}{$\begin{array}{c}\text { Total } \\
(\mathrm{n}=465)\end{array}$}} & \multicolumn{4}{|c|}{ Grupo } & \multirow{3}{*}{$\mathbf{t}$} & \multirow{3}{*}{ p } \\
\hline & & & \multicolumn{2}{|c|}{$\begin{array}{c}\text { Intervenção } \\
(n=224)\end{array}$} & \multicolumn{2}{|c|}{$\begin{array}{l}\text { Controle } \\
(n=241)\end{array}$} & & \\
\hline & $\mathbf{n}$ & $\%$ & $\mathbf{n}$ & $\%$ & $\mathbf{n}$ & $\%$ & & \\
\hline Idade (anos) & & & & & & & $-1,265$ & 0,237 \\
\hline Média (DP) & \multicolumn{2}{|c|}{$24,7(6,0)$} & \multicolumn{2}{|c|}{$24,3(5,7)$} & \multicolumn{2}{|c|}{$25,0(6,2)$} & & \\
\hline Mínimo-Máximo & \multicolumn{2}{|c|}{$18-55$} & \multicolumn{2}{|c|}{$18-50$} & \multicolumn{2}{|c|}{$18-55$} & & \\
\hline Sexo & & & & & & & 2,729 & 0,099 \\
\hline Masculino & 300 & 64,5 & 136 & 60,7 & 164 & 68,0 & & \\
\hline Feminino & 165 & 35,5 & 88 & 39,3 & 77 & 32,0 & & \\
\hline ABEP & & & & & & & 3,744 & 0,852 \\
\hline A & 139 & 29,9 & 68 & 30,4 & 71 & 29,5 & & \\
\hline $\mathrm{B}$ & 261 & 56,1 & 123 & 54,9 & 138 & 57,2 & & \\
\hline$C, D$ ou $E$ & 65 & 14,0 & 33 & 14,7 & 32 & 13,3 & & \\
\hline Escore AUDIT & & & & & & & $-1,050$ & 0,332 \\
\hline Média (DP) & \multicolumn{2}{|c|}{$12,6(4,1)$} & \multicolumn{2}{|c|}{$12,4(4,0)$} & \multicolumn{2}{|c|}{$12,8(4,2)$} & & \\
\hline Mínimo-Máximo & \multicolumn{2}{|c|}{$8-33$} & \multicolumn{2}{|c|}{$8-27$} & \multicolumn{2}{|c|}{$8-33$} & & \\
\hline AUDIT & & & & & & & 0,940 & 0,639 \\
\hline Uso de risco & 333 & 71,6 & 164 & 73,2 & 169 & 70,1 & & \\
\hline Uso nocivo & 100 & 21,5 & 47 & 21,0 & 53 & 22,0 & & \\
\hline Dependência & 32 & 6,9 & 13 & 5,8 & 19 & 7,9 & & \\
\hline
\end{tabular}

DP: desvio padrão; t: valor de qui-quadrado ou teste t; ABEP: Associação Brasileira de Estudos Populacionais;

AUDIT: Alcohol Use Disorders Identification Test 
Tabela 2. Distribuição dos 465 frequentadores de baladas de acordo com resposta ao acompanhamento de 6 meses.

\begin{tabular}{|c|c|c|c|c|c|c|c|c|}
\hline \multirow[t]{2}{*}{ Variável } & \multicolumn{2}{|c|}{$\begin{array}{c}\text { Total } \\
(n=465)\end{array}$} & \multicolumn{2}{|c|}{$\begin{array}{l}\text { Participantes } \\
\qquad(\mathrm{n}=156)\end{array}$} & \multicolumn{2}{|c|}{$\begin{array}{c}\text { Perda } \\
(\mathrm{n}=309)\end{array}$} & \multirow[t]{2}{*}{$t$} & \multirow[t]{2}{*}{$p$} \\
\hline & $\mathbf{n}$ & $\%$ & $\mathbf{n}$ & $\%$ & $\mathbf{n}$ & $\%$ & & \\
\hline Grupo & & & & & & & 0,573 & 0,449 \\
\hline Intervenção & 224 & 48,2 & 79 & 50,6 & 145 & 46,9 & & \\
\hline Controle & 241 & 51,8 & 77 & 49,4 & 164 & 53,1 & & \\
\hline Idade (anos) & & & & & & & 0,171 & 0,865 \\
\hline Média (DP) & \multicolumn{2}{|c|}{$24,7(6,0)$} & \multicolumn{2}{|c|}{$24,7(5,4)$} & \multicolumn{2}{|c|}{$24,6(6,2)$} & & \\
\hline Mínimo-Máximo & \multicolumn{2}{|c|}{$18-55$} & \multicolumn{2}{|c|}{$18-50$} & \multicolumn{2}{|c|}{$18-55$} & & \\
\hline Sexo & & & & & & & 0,005 & 0,942 \\
\hline Masculino & 300 & 64,5 & 101 & 64,7 & 199 & 64,4 & & \\
\hline Feminino & 165 & 35,5 & 55 & 35,3 & 110 & 35,6 & & \\
\hline ABEP & & & & & & & 0,954 & 0,621 \\
\hline A & 139 & 29,9 & 51 & 32,7 & 88 & 28,5 & & \\
\hline B & 261 & 56,1 & 85 & 54,5 & 176 & 57,0 & & \\
\hline C, D ou E & 65 & 14,0 & 20 & 12,8 & 45 & 14,5 & & \\
\hline Escore AUDIT $_{\text {inicial }}$ & & & & & & & $-0,678$ & 0,542 \\
\hline Média (DP) & \multicolumn{2}{|c|}{$12,6(4,1)$} & \multicolumn{2}{|c|}{$12,4(4,1)$} & \multicolumn{2}{|c|}{$12,7(4,1)$} & & \\
\hline Mínimo-Máximo & \multicolumn{2}{|c|}{$8-33$} & \multicolumn{2}{|c|}{$8-27$} & \multicolumn{2}{|c|}{$8-33$} & & \\
\hline $\mathrm{AUDIT}_{\text {inicial }}$ & & & & & & & 0,111 & 0,940 \\
\hline Uso de risco & 333 & 71,6 & 113 & 72,5 & 220 & 71,2 & & \\
\hline Uso nocivo & 100 & 21,5 & 33 & 21,1 & 67 & 21,7 & & \\
\hline Dependência & 32 & 6,9 & 10 & 6,4 & 22 & 7,1 & & \\
\hline
\end{tabular}

DP: desvio padrão; t: valor de qui-quadrado ou teste t; ABEP: Associação Brasileira de Estudos Populacionais; AUDIT $_{\text {inicial }}$ : Alcohol Use Disorders Identification Test inicial

Tabela 3. Comparações entre grupos nos dois tempos e efeito da intervenção em 6 meses (interação grupo e tempo) para análise ITT e LOCF.

\begin{tabular}{|c|c|c|c|c|c|c|c|c|c|c|c|c|c|c|}
\hline \multirow{3}{*}{ Variável } & \multicolumn{6}{|c|}{ Grupos } & \multirow{2}{*}{\multicolumn{4}{|c|}{ Comparação entre grupos ${ }^{a}$}} & \multirow{2}{*}{\multicolumn{4}{|c|}{ Efeito intervenção em 6 meses ${ }^{b}$}} \\
\hline & \multicolumn{3}{|c|}{$\begin{array}{c}\text { Intervenção } \\
(\mathbf{n}=\mathbf{2 2 4})\end{array}$} & \multicolumn{3}{|c|}{$\begin{array}{l}\text { Controle } \\
(\mathrm{n}=241)\end{array}$} & & & & & & & & \\
\hline & n & $\%$ & IC95\% & n & $\%$ & IC95\% & $\mathrm{OR}_{\mathrm{b}}{ }^{\mathrm{a}}$ & IC95\% & $\mathrm{OR}_{\mathrm{aj}}^{\mathrm{b}}$ & IC95\% & $\begin{array}{c}\mathrm{OR}_{\mathrm{b}}{ }^{\mathrm{a}} \\
(\mathrm{IC} \mathrm{C} \% \mathrm{~F})\end{array}$ & $\mathbf{p}$ & $\begin{array}{c}\mathrm{OR}_{\mathrm{aj}}^{\mathrm{b}} \\
(\mathrm{IC} 95 \%)\end{array}$ & p \\
\hline
\end{tabular}

Intenção de tratamento - ITT

\begin{tabular}{|c|c|c|c|c|c|c|c|c|c|c|c|c|c|c|}
\hline \multicolumn{15}{|l|}{$\mathrm{BD}_{\text {mês }}$} \\
\hline Início & 161 & 71,9 & $66,0-77,8$ & 183 & 75,9 & $70,5-81,3$ & 0,81 & $0,53-1,23$ & 0,79 & $0,52-1,20$ & 0,97 & \multirow{2}{*}{0,930} & \multirow{2}{*}{$\begin{array}{c}0,98 \\
(0,49-1,98)\end{array}$} & \multirow{2}{*}{0,959} \\
\hline 6 meses & 51 & 63,9 & $53,3-73,8$ & 54 & 69 & $59,0-79,0$ & 0,78 & $0,41-1,49$ & 0,77 & $0,40-1,48$ & $(0,48-1,94)$ & & & \\
\hline \multicolumn{15}{|l|}{$\mathrm{BD}_{\text {semana }}$} \\
\hline Início & 119 & 53,4 & $46,5-59,9$ & 137 & 56,8 & $50,6-63,1$ & 0,87 & $0,60-1,25$ & 0,91 & $0,63-1,31$ & & \multirow{2}{*}{0,795} & \multirow{2}{*}{$\begin{array}{c}0,92 \\
(0,51-1,67)\end{array}$} & \multirow{2}{*}{0,794} \\
\hline 6 meses & 32 & 41,8 & $31,8-51,8$ & 34 & 47,2 & $37,0-57,4$ & 0,80 & $0,45-1,44$ & 0,84 & $0,46-1,51$ & $(0,52-1,66)$ & & & \\
\hline \multicolumn{15}{|c|}{ Falta controle } \\
\hline Início & 111 & 49,8 & $43,2-56,3$ & 120 & 49,8 & $43,5-56,1$ & 0,99 & $0,69-1,44$ & 1,01 & $0,69-1,45$ & & \multirow{2}{*}{0,062} & \multirow{2}{*}{$\begin{array}{c}0,55 \\
(0,30-1,02)\end{array}$} & \multirow{2}{*}{0,058} \\
\hline 6 meses & 35 & 43,9 & $33,6-54,2$ & 49 & 58,4 & $48,1-68,7$ & 0,56 & $0,30-1,01$ & 0,55 & $0,30-1,01$ & $(0,30$ & & & \\
\hline \multicolumn{15}{|c|}{ Last Observed Carried Forward - LOCF } \\
\hline \multicolumn{15}{|l|}{$\mathrm{BD}_{\text {mês }}$} \\
\hline Início & 161 & 71,9 & $66,0-77,8$ & 183 & 75,9 & $70,5-81,3$ & 0,81 & $0,53-1,23$ & 0,79 & $0,52-1,20$ & & \multirow{2}{*}{0,901} & \multirow{2}{*}{$\begin{array}{c}0,99 \\
(0,74-1,31)\end{array}$} & \multirow{2}{*}{0,928} \\
\hline 6 meses & 161 & 68,3 & $62,2-74,4$ & 183 & 73 & $67,4-78,6$ & 0,80 & $0,53-1,19$ & 0,78 & $0,52-1,16$ & $(0,75-1,29)$ & & & \\
\hline \multicolumn{15}{|l|}{$\mathrm{BD}_{\text {semana }}$} \\
\hline Início & 119 & 53,4 & $46,8-59,9$ & 137 & 56,8 & $50,6-63,1$ & 0,87 & $0,60-1,25$ & 0,91 & $0,62-1,31$ & 0,94 & \multirow{2}{*}{0,591} & \multirow{2}{*}{$\begin{array}{c}0,94 \\
(0,75-1,17)\end{array}$} & \multirow{2}{*}{0,580} \\
\hline 6 meses & 111 & 49,8 & $43,2-56,3$ & 132 & 54,8 & $48,5-61,0$ & 0,82 & $0,57-1,18$ & 0,85 & $0,59-1,23$ & $(0,76-1,17)$ & & & \\
\hline \multicolumn{15}{|c|}{ Falta controle } \\
\hline Início & 111 & 49,8 & $43,2-56,3$ & 120 & 49,8 & $43,5-56,1$ & 0,99 & $0,69-1,44$ & 1,00 & $0,69-1,45$ & 0,90 & \multirow{2}{*}{0,352} & 0,90 & \multirow{2}{*}{0,352} \\
\hline 6 meses & 106 & 47,5 & $41,0-54,1$ & 121 & 50,2 & $43,9-56,5$ & 0,90 & $0,62-1,29$ & 0,90 & $0,62-1,30$ & $(0,72-1,12)$ & & $(0,71-1,13)$ & \\
\hline
\end{tabular}

ITT: intention to treat analysis; LOCF: last observed carried forward; $\mathrm{BD}_{\text {mês }}$ : binge drinking no mês; $\mathrm{BD}_{\text {semana }}$ : binge drinking na semana

a Referência: grupo controle.

${ }^{\mathrm{b}}$ Modelo linear generalizado com procedimento xtlogit do Stata ajustado pelo efeito de grupo, tempo, grupo*tempo, sexo, idade e ABEP.

Referência: grupo controle. 


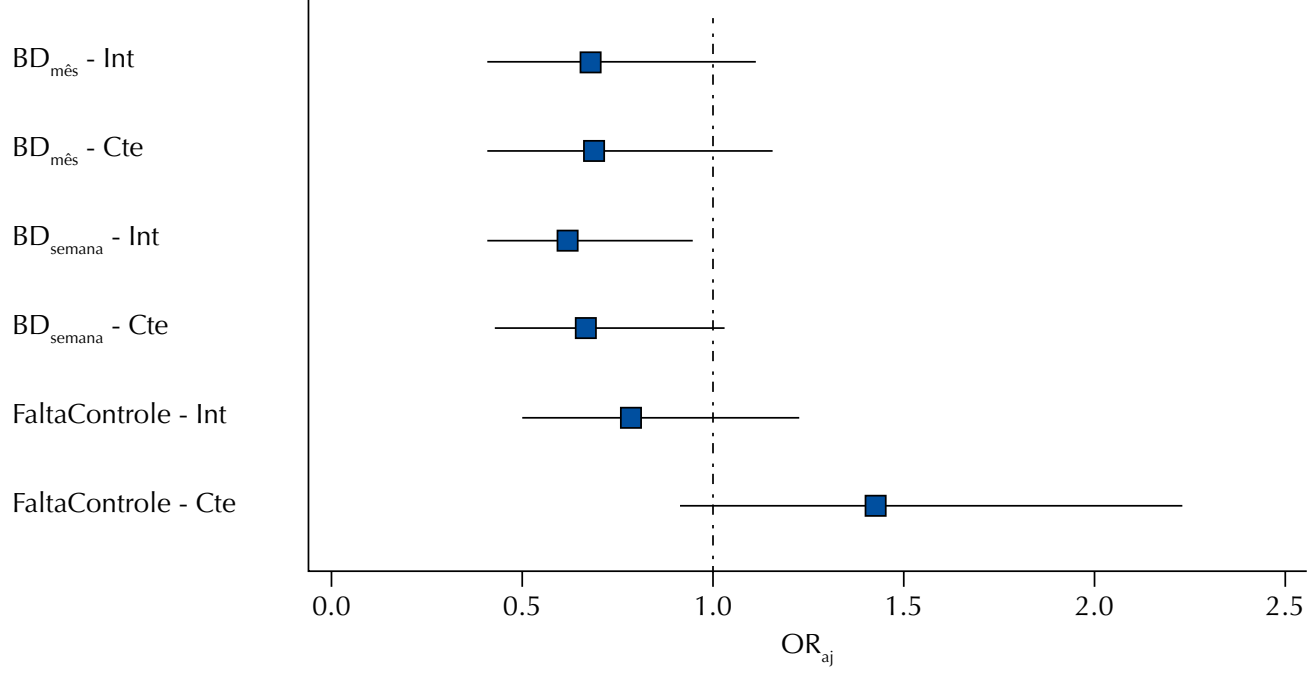

$\mathrm{BD}_{\text {mês: }}$ episódio de binge drinking nos últimos 30 dias (mês); $\mathrm{BD}_{\text {semana }}$ : episódio de binge drinking nos últimos 7 dias (semana); Int: Intervenção; Cte: Controle; FaltaControle: Falta de controle sobre o comportamento de beber (falta de controle); $\mathrm{OR}_{\mathrm{a}}$ : odds ratio obtido por modelo linear generalizado com procedimento $x$ tlogit do Stata ajustado por grupo, tempo, grupo*tempo, sexo, idade e classe socioeconômica. Os valores de OR representados têm como referência o tempo inicial (momento 0 )

Figura 2. Forest plot para a comparação de mudanças de proporção intragrupo, comparando-se os momentos início (0) e 6 meses de acompanhamento, para cada desfecho avaliado. $(n=465)$

Também não foi observado efeito significativo da intervenção em seis meses de acompanhamento em nenhum dos desfechos avaliados. Entretanto, os valores de OR do efeito de intervenção em seis meses dos desfechos avaliados são menores do que 1, mostrando que, em comparação ao grupo controle, o grupo intervenção apresentou um decréscimo no $\mathrm{BD}_{\text {mês }}, \mathrm{BD}_{\text {semana }} \mathrm{e}$ falta de controle. Além disso, nota-se resultado marginalmente significante $(\mathrm{p}=0,058)$ para o efeito da intervenção na redução da prevalência de relato de falta de controle sobre o comportamento de beber $\left(\mathrm{OR}_{\mathrm{aj}}=0,55 ; \mathrm{IC} 95 \% 0,30-1,02\right)$. No entanto, quando os dados são imputados via LOCF, a tendência de significância para o efeito da intervenção sobre a falta de controle de beber deixa de existir.

A Figura 2 apresenta a comparação dos desfechos no tempo para cada grupo avaliado. Houve efeito significativo do grupo intervenção para $\mathrm{BD}_{\text {semana }}$, ou seja, verificou-se, no sexto mês de acompanhamento, uma redução de $38 \%(\mathrm{p}=0,026)$ na prática de binge drinking na semana, após ajuste por sexo, idade e classe socioeconômica, quando comparado ao momento inicial; para o grupo controle, para esse mesmo desfecho, não se observou efeito significativo $(\mathrm{p}=0,062)$.

\section{DISCUSSÃO}

Os resultados aqui apresentados fazem parte da primeira pesquisa epidemiológica no Brasil sobre comportamento de risco em baladas da cidade de São Paulo. O estudo investigou o efeito, após seis meses, de uma intervenção on-line para a redução da prática do BD em frequentadores de balada da cidade de São Paulo. O principal achado deste estudo foi a redução no relato de $\mathrm{BD}_{\text {semana }}$ em $38 \%$ após seis meses da exposição à intervenção. O grupo com uso problemático de álcool representou $44 \%$ do entrevistados nas baladas da cidade e, dessa forma, necessitam de intervenção imediata ou de tratamento ${ }^{30}$. Os resultados ainda mostraram que a maioria dos sujeitos que optaram por participar do ECR era homem $(64,5 \%)$ e pertencia às classes socioeconômicas A ou B (86\%). A média de idade era de 24,7 anos.

Os dados sociodemográficos dos participantes da intervenção corroboram Siliquini et al. ${ }^{7}$, que encontram em baladas de seis países europeus uma proporção de 67,9\% de homens, com idade entre 20 e 24 anos e alta escolaridade, sugerindo uma boa classe socioeconômica. Outro estudo 
brasileiro, realizado em Belo Horizonte, MG, com 913 pessoas que tinham saído de ambientes de lazer como bares e baladas, constatou uma população de $80 \%$ do sexo masculino, com faixa etária entre 18 e 30 anos e renda familiar superior a oito salários mínimos ${ }^{35}$.

Intervenções digitais com base em feedback normativo personalizados são mais comumente aplicadas a estudantes universitários ${ }^{13}$. Kypri et al..$^{19}$, em um estudo com 2.435 universitários neozelandeses que faziam uso problemático de álcool de acordo com a classificação AUDIT, encontraram redução de $9 \%$ na frequência de beber, de $6 \%$ da quantidade bebida e de $14 \%$ do volume consumido, após seis meses da exposição a uma intervenção digital de feedback normativo personalizado. Por outro lado, em uma revisão sistemática, Foxcroft et al. ${ }^{36}$ avaliaram 66 estudos buscando determinar a redução das consequências danosas do abuso de álcool em estudantes por meio de feedback normativo. Não se encontrou benefícios significativos associados às intervenções com normas sociais nessa população, mas os autores ressaltam a dificuldade de se comparar esses estudos devido à heterogeneidade de desenho ${ }^{36}$.

Na cidade de Curitiba, no Paraná, um estudo com a população universitária comparando intervenções digitais com intervenções não computadorizadas (baseadas em entrevistas motivacionais) constatou resultados mais positivos nas intervenções digitais para redução do uso de álcool, recomendando essa alternativa frente às entrevistas pessoais, pois são facilmente acessadas por estudantes e abrangem um número maior de participantes ${ }^{24}$.

Esse tipo de ferramenta parece ter efeitos positivos quando se analisa a população geral. Em uma revisão sistemática, Tait e Christensen ${ }^{9}$ analisaram 14 estudos $(\mathrm{n}=7.082)$ a respeito de intervenções na web para jovens com problemas com o uso de álcool e constatam uma redução de $12 \%$ da quantidade de álcool consumida, 35\% na frequência de BD e 57\% nas consequências negativas do uso de álcool nos diferentes estudos. No Brasil, Andrade et al. ${ }^{23}$ também analisaram ferramentas digitais na população geral em um estudo com 929 participantes. Os autores destacam que, apesar da relativa baixa aderência ao estudo, pode-se reduzir o consumo de álcool após seis semanas em $62,5 \%$ dos usuários com consumo danosos de álcool, ressaltando a vantagem de as ferramentas estarem disponíveis para populações remotas e a qualquer hora do dia.

A principal vantagem para o uso de ferramentas digitais é a acessibilidade, principalmente entre jovens universitários ${ }^{16}$, população que compõe em grande parte o cenário das baladas paulistanas ${ }^{32}$, além do custo-efetividade e praticidade da ferramenta ${ }^{15}$. Para Simon-Arndt et al., que aplicaram o mesmo tipo de intervenção em marinheiros dos Estados Unidos, outra grande vantagem do método era a questão da privacidade fornecida pela internet quando comparada a ações presenciais ${ }^{17}$.

No presente estudo, houve uma redução significativa da prática de BD na semana estimada em $38 \%$ entre os participantes do grupo intervenção após seis meses, não observada no grupo controle. No entanto, quando avaliado o efeito do programa (interação entre grupo e tempo), os resultados para BD não são significativos e a redução da falta de controle é marginalmente significante, apesar de todos os odds ratios mostrarem tendência protetora da intervenção. A opção mais severa, do ponto de vista estatístico, de se utilizar abordagem de LOCF, considerando no extremo que as perdas mantiveram seu padrão de consumo do tempo inicial, sugere que não há efeito da intervenção em nenhum dos desfechos avaliados. Dessa forma, não é adequado afirmar categoricamente que a referida intervenção é efetiva nesse cenário, já que, quando incluímos as perdas nas análises mediante o pior cenário possível (não mudança), houve perda completa da significância.

Dentre as limitações deste estudo, estão: a taxa de perda de sujeitos nas diferentes etapas; a dificuldade de acesso à internet de alguns frequentadores de balada, limitando a amplitude do ECR; e a impossibilidade de comparar nossos resultados com os de outros estudos, devido ao caráter inédito do uso de intervenções digitais em frequentadores de baladas. Outra limitação se refere à adaptação quanto ao critério de BD para mulheres, visto que, neste estudo, considerou-se BD cinco ou mais doses para ambos os sexos, enquanto diversos estudos 
optam por reduzir o corte para mulheres, para quatro doses. Mesmo com essas limitações, reforça-se o caráter inovador deste estudo no Brasil, um país marcado por grandes danos advindos do abuso de álcool pela sua população.

São necessários mais estudos que avaliem esta e outras metodologias que reduzam a prevalência e as consequências do abuso de álcool, tendo em vista a impossibilidade de concluir sobre a eficácia das ferramentas de intervenção digital na redução de BD entre frequentadores de baladas.

\section{REFERÊNCIAS}

1. Calafat A, Koller M; Comisión de las Comunidades Europeas. Enjoying the nightlife in Europe: the role of moderation. Palma de Mallorca: IREFREA; 2003.

2. Graham K, Bernards S, Osgood DW, Wells S. Bad nights or bad bars? Multi-level analysis of environmental predictors of aggression in late-night large-capacity bars and clubs. Addiction. 2006;101(11):1569-80. https://doi.org/10.1111/j.1360-0443.2006.01608.x

3. Reingle J, Thombs DL, Weiler RM, Dodd VJ, O'Mara R, Pokorny SB. An exploratory study of bar and nightclub expectancies. J Am Coll Health. 2009;57(6):629-38. https://doi.org/10.3200/JACH.57.6.629-638

4. Demant J, Heinskou MB. Taking a chance: sex, alcohol and acquaintance rape. Young. 2011;19(4):397-415. https:/doi.org/10.1177/110330881101900403

5. Calafat A, Blay NT, Hughes K, Bellis M, Juan M, Duch M, Kokkevi A. Nightlife young risk behaviours in Mediterranean versus other European cities: are stereotypes true? Eur J Public Health. 2011;21(3):311-5. https://doi.org/10.1093/eurpub/ckq141

6. National Institute of Alcohol Abuse and Alcoholism. NIAAA Council approves definition of binge drinking. NIAAA Newslett. 2004 Winter [citado 3 jul 2017];(3):3. Disponível em: https:// pubs.niaaa.nih.gov/publications/Newsletter/winter2004/Newsletter_Number3.pdf

7. Siliquini R, Colombo A, Berchialla P, Bert F, TEND-D Group. Binge drinking and psychoactive drug use in a cohort of European youths. J Public Health Res. 2012;1(1):83-8. https://doi.org/10.4081/jphr.2012.e15

8. Naimi TS, Brewer RD, Mokdad A, Denny C, Serdula MK, Marks JS. Binge drinking among US adults. JAMA. 2003;289(1):70-5. https://doi.org/10.1001/jama.289.1.70

9. Tait RJ, Christensen H. Internet-based interventions for young people with problematic substance use: a systematic review. Med J Aust. 2010 [citado 3 jul 2017];192(11 Suppl):S15-21. Disponível em: https://www.mja.com.au/system/files/issues/192_11_070610/tai10832_fm.pdf

10. Rooke S, Thorsteinsson E, Karpin A, Copeland J, Allsop D. Computer-delivered interventions for alcohol and tobacco use: a meta-analysis. Addiction. 2010;105(8):1381-90. https://doi.org/10.1111/j.1360-0443.2010.02975.x

11. Kypri K, Langley JD. Perceived social norms and their relation to university student drinking. J Stud Alcohol. 2003;64(6):829-34. https://doi.org/10.15288/jsa.2003.64.829

12. McAlaney J, Bewick B, Hughes C. The international development of the 'Social Norms' approach to drug education and prevention. Drugs (Abingdon Engl). 2011;18(2):81-9. https://doi.org/10.3109/0968787631003610977

13. Walters ST, Neighbors C. Feedback interventions for college alcohol misuse: what, why and for whom? Addict Behav. 2005;30(6):1168-82. https://doi.org/10.1016/j.addbeh.2004.12.005

14. Palfai TP, Winter M, Lu J, Rosenbloom D, Saitz R. Personalized feedback as a universal prevention approach for college drinking: a randomized trial of an e-mail linked universal web-based alcohol intervention. J Prim Prev. 2014;35(2):75-84. https://doi.org/10.1007/s10935-013-0337-9

15. Riper H, Straten A, Keuken M, Smit F, Schippers G, Cuijpers P. Curbing problem drinking with personalized-feedback interventions: a meta-analysis. Am J Prev Med. 2009;36(3):247-55. https://doi.org/10.1016/j.amepre.2008.10.016

16. White A, Kavanagh D, Stallman H, Klein B, Kay-Lambkin F, Proudfoot J, et al. Online alcohol interventions: a systematic review. J Med Internet Res. 2010;12(5):e62. https://doi.org/10.2196/jmir.1479 
17. Simon-Arndt CM, Hurtado SL, Patriarca-Troyk LA. Acceptance of web-based personalized feedback: user ratings of an alcohol misuse prevention program targeting U.S. Marines. Health Commun. 2006;20(1):13-22. https://doi.org/10.1207/s15327027hc2001_2

18. Lewis MA, Patrick ME, Litt DM, Atkins DC, Kim T, Blayney JA, et al. Randomized controlled trial of a web-delivered personalized normative feedback intervention to reduce alcohol-related risky sexual behavior among college students. J Consult Clin Psychol. 2014;82(3):429-40. https://doi.org/10.1037/a0035550

19. Kypri K, Hallett J, Howat P, McManus A, Maycock B, Bowe S, Horton NJ. Randomized controlled trial of proactive web-based alcohol screening and brief intervention for university students. Arch Intern Med. 2009;169(16):1508-14. https://doi.org/10.1001/archinternmed.2009.249

20. Kypri K, Langley JD, Saunders JB, Cashell-Smith ML, Herbison P. Randomized controlled trial of web-based alcohol screening and brief intervention in primary care. Arch Intern Med. 2008;168(5):530-6. https://doi.org/10.1001/archinternmed.2007.109

21. Kypri K, Saunders JB, Williams SM, McGee RO, Langley JD, Cashell-Smith ML, et al. Web-based screening and brief intervention for hazardous drinking: a double-blind randomized controlled trial. Addiction. 2004;99(11):1410-7. https://doi.org/10.1111/j.1360-0443.2004.00847.x

22. Instituto Brasileiro de Geografia e Estatística. Pesquisa Nacional por Amostra de Domicílios 2014. Rio de Janeiro: IBGE; 2014 [citado 3 jul 2017]. Disponível em: http://www.ibge.gov.br/ home/estatistica/populacao/trabalhoerendimento/pnad2014/default.shtm

23. Andrade AL, Lacerda RB, Gomide HP, Ronzani TM, Sartes LM, Martins LF, et al. Web-based self-help intervention reduces alcohol consumption in both heavy-drinking and dependent alcohol users: a pilot study. Addict Behav. 2016;63:63-71. https://doi.org/10.1016/j.addbeh.2016.06.027

24. Christoff AO, Boerngen-Lacerda R. Reducing substance involvement in college students: a three-arm parallel-group randomized controlled trial of a computer-based intervention. Addict Behav. 2015;45:164-71. https://doi.org/10.1016/j.addbeh.2015.01.019

25. Warpenius K, Holmila M, Mustonen $\mathrm{H}$. Effects of a community intervention to reduce the serving of alcohol to intoxicated patrons. Addiction. 2010;105(6):1032-40. https://doi.org/10.1111/j.1360-0443.2009.02873.x

26. Voas RB, Furr-Holden D, Lauer E, Bright K, Johnson MB, Miller B. Portal surveys of time-out drinking locations: a tool for studying binge drinking and AOD use. Eval Rev. 2006;30(1):44-65. https://doi.org/10.1177/0193841X05277285.

27. Carlini C, Andreoni S, Martins SS, Benjamin M, Sanudo A, Sanchez ZM. Environmental characteristics associated with alcohol intoxication among patrons in Brazilian nightclubs. Drug A/cohol Rev. 2014;33(4):358-66. https://doi.org/10.1111/dar.12155

28. Sanchez ZM, Ribeiro KJ, Wagner GA. Binge drinking associations with patrons' risk behaviors and aAlcohol effects after leaving a nightclub: sex differences in the "Balada com Ciência" Portal Survey Study in Brazil. PLoS One. 2015;10(8):e0133646. https://doi.org/10.1371/journal.pone.0133646

29. Santos R, Baldin Y, Carlini CM, Sanchez ZM. Classification of alcohol use disorders among nightclub patrons: associations between high-risk groups, sociodemographic factors and illicit drug use. Am J Drug Alcohol Abuse. 2015;41(5):433-41. https://doi.org/10.3109/00952990.2015.1050497

30. Babor TF, Higgins-Biddle JC, Saunders JB, Monteiro MG. AUDIT: The Alcohol Use Disorders Identification Test: Guidelines for Use in Primary Care. 2.ed. Geneva: WHO Department of Mental Health and Substance Dependence; 2001 [citado 14 jul 2017]. Disponível em: http:// apps.who.int/iris/bitstream/10665/67205/1/WHO_MSD_MSB_01.6a.pdf

31. Reinert DF, Allen JP. The alcohol use disorders identification test: an update of research findings. Alcohol Clin Exp Res. 2007;31(2):185-99. https://doi.org/10.1111/j.1530-0277.2006.00295.x

32. Santos MGR, Paes AT, Sanudo A, Sanchez ZM. Factors associated with pre-drinking among nightclub patrons in the city of São Paulo. Alcohol Alcohol. 2015;50(1):95-102. https://doi.org/10.1093/alcalc/agu055

33. Associação Brasileira de Empresas de Pesquisa. Critério de Classificação Econômica no Brasil. Rio de Janeiro: ABEP; 2012.

34. Laranjeira R, Pinsky I, Sanches M, Zaleski M, Caetano R. Alcohol use patterns among Brazilian adults. Rev Bras Psiquiatr. 2010;32(3):231-41. https://doi.org/10.1590/S1516-44462009005000012 
35. Campos VR, Salgado R, Rocha MC, Duailibi S, Laranjeira R. Prevalência do beber e dirigir em Belo Horizonte, Minas Gerais, Brasil. Cad Saude Publica. 2008;24(4):829-34. https://doi.org/10.1590/S0102-311X2008000400013

36. Foxcroft DR, Moreira MT, Almeida Santimano NM, Smith LA. Social norms information for alcohol misuse in university and college students. Cochrane Database Syst Rev. 2015;(12):CD006748. https://doi.org/10.1002/14651858.CD006748.pub4

Financiamento: Fundação de Amparo à Pesquisa do Estado de São Paulo (Fapesp - Processos 11/51658-0 e 14/18602-0).

Contribuição dos Autores: YCB: análise e interpretação dos dados, elaboração e revisão do manuscrito. AS: concepção e planejamento da análise, interpretação dos dados e revisão do manuscrito. ZMS: concepção e planejamento do estudo, coleta, análise e interpretação dos dados, revisão do manuscrito, aprovação da versão final, responsabilidade pública pelo conteúdo do artigo.

Conflito de Interesses: Os autores declaram não haver conflito de interesses. 